\title{
Different loading times for dental implants - no clinically important differences?
}

\author{
Abstracted from \\ Esposito M, Grusovin MG, Maghaireh H, Worthington HV. \\ Interventions for replacing missing teeth: different times for loading dental implants. \\ Cochrane Database Syst Rev 2013; Issue 3. Art. No.: CD003878. \\ Address for correspondence: Luisa Fernandez Mauleffinch, Review Group Co-ordinator, \\ Cochrane Oral Health Group, MANDEC, School of Dentistry, University of Manchester, \\ Higher Cambridge Street, Manchester, M15 6FH, UK. E-mail: luisa.fernandez@manchester.ac.uk
}

\section{Question: What is the effect of different loading times on the outcomes of implant placement?}

Data sources The Cochrane Oral Health Group's Trials Register, the Cochrane Central Register of Controlled Trials (CENTRAL), Medline, and Embase databases were searched. Reference lists of identified articles were also scanned for relevant papers. There were no restrictions on language or date of publication.

Study selection Randomised controlled trials (RCTs) of parallel group design and of split-mouth design including root-form osseointegrated dental implants having a follow-up of four months to one year after loading were included.

Data extraction and synthesis Data were independently extracted, in duplicate, by at least two review authors. The outcome measures were prosthesis and implant failures and radiographic marginal bone level changes. Risk of bias was assessed for each trial by at least two review authors. Results were combined using fixed-effect models with mean differences (MD) for continuous outcomes and risk ratios (RR) for dichotomous outcomes with $95 \%$ confidence intervals (CI).

Results Twenty-six trials involving a total of 1217 participants and 2120 implants were included. The risk of bias was low in three trials, high in 12 and unclear for the remaining eleven. In nine studies there were no prosthetic failures within the first year, with no implant failures in seven studies and the mean rate of implant failure in all 26 trials was a low $2.5 \%$. From 15 RCTs comparing immediate with conventional loading there was no evidence of a difference in either prosthesis failure (RR $1.87 ; 95 \% \mathrm{Cl} 0.70$ to $5.01 ; 8$ trials) or implant failure (RR $1.65 ; 95 \% \mathrm{Cl} 0.68$ to $3.98 ; 10$ trials) in the first year. However, there is some evidence of a small reduction in bone loss favouring immediate loading (MD $-0.10 \mathrm{~mm} ; 95 \% \mathrm{Cl}-0.20$ to $-0.01 ; \mathrm{P}=0.03 ; 9$ trials), but this very small difference may not be clinically important. From three RCTs which compared early loading with conventional loading, there is insufficient evidence to determine whether or not there is a clinically important difference in prosthesis failure, implant failure or bone loss. Six RCTs compared immediate and early loading and found insufficient evidence to determine

This paper is based on a Cochrane Review published in the Cochrane Library 2013, issue 3 (see www.thecochranelibrary.com for information). Cochrane Reviews are regularly updated as new evidence emerges and in response to feedback, and the Cochrane Library should be consulted for the most recent version of the review. whether or not there is a clinically important difference in prosthesis failure, implant failure or bone loss. From the two trials that compared occlusal loading with non-occlusal loading there is insufficient evidence to determine whether there is a clinically important difference in the outcomes of prosthesis failure, implant failure or bone loss. No trials were identified which evaluated progressive loading of implants.

Conclusions Overall there was no convincing evidence of a clinically important difference in prosthesis failure, implant failure or bone loss associated with different loading times of implants. More welldesigned RCTs are needed and should be reported according to the CONSORT guidelines.

\section{Commentary}

For many dental practitioners, deciding when to load an implant fixture is one of the more perplexing questions surrounding the restorative phase of implant treatment. Since Branemark et al. ${ }^{1}$ first proposed the two-stage surgical protocol during which the implant fixture is kept load-free during the healing period, loading protocols have been a central focus of discussion resulting in a variety of opinions. The conventional Branemark protocol has enjoyed a high degree of long-term clinical success in both partially and fully edentulous patients; however, most would agree that it would be beneficial to know whether or not the healing period could be shortened without jeopardising the osseointegration of the implant.

This Cochrane Review revisits the question of whether or not there is a difference in success rates between immediately or earlyloaded implants compared with conventionally loaded implants. In 2003 the authors' initial Cochrane Database Systematic Review on this subject was published, with subsequent updates in 2007, 2008, 2009, and most recently, in 2013. This latest version reexamines the current state of the evidence and offers considerably more insight than their relatively limited 2003 review, while providing a more refined conclusion to that of the 2009 review.

Only three RCTs that met the selection criteria were identified in the initial 2003 review and only two of these trials, with a total of 68 patients, were suitable for inclusion. Understandably, they concluded that according to the available research at the time, it was unknown as to how predictable the approach of immediately loading implants would be. ${ }^{2}$ By the time of the 2009 review, there were twenty RCTs that met the selection criteria with 300 participants and 790 implants available for analysis. Their findings stated that it was possible to load 
an implant immediately or early in selected patients, although not all clinicians would necessarily achieve optimal results. ${ }^{3}$ In attempting to answer the question of whether or not the healing period could be shortened without jeopardising the osseointegration of the implant, their thorough review led to a conclusion that was still less definitive than a practitioner might hope to see.

As would be expected, the authors had more data at their disposal for the 2013 review, and as such, their findings are slightly more conclusive, however the authors are clear in stating that there is still insufficient information to draw definitive conclusions. Forty-five RCTs were identified as meeting the selection criteria and out of these, 26 trials having 1217 participants and 2120 implants, were chosen to be analysed in the review. Similar to the 2009 review, the three fundamental outcomes measured were prosthesis failure, implant failure and radiographic marginal bone level changes after four months to one year from loading. The findings between the two differ in that the newer review showed that there was no convincing evidence of a clinically important difference in the failure rate based on loading time, while the 2009 review found that there was some evidence that immediate or early loading may have a slightly poorer outcome than conventional loading.

The authors included all of the randomised trials available to date, but they concluded that the low failure rate of both implants and prostheses in each of these trials meant that it was not statistically possible to determine whether or not there was any important difference between different loading times. They also point out that research suggests that thousands of participants would need to be included in RCTs in order to produce the conclusive evidence we practitioners desire. ${ }^{4}$ In the short term at least, this is unlikely to occur.
Over the course of the last 15 years the authors have made significant scholarly contributions in their effort to answer questions related to failure of implants. Their conclusions in this comprehensive review bring us one step closer to understanding the benefits and risks of loading times for dental implants.

Gary L Stafford

Department of General Dental Sciences, Marquette University School of Dentistry, Milwaukee, Wisconsin, USA

\section{Practice points}

- The risk of implant failure can be substantially minimised by proper patient selection, well-trained operators and achieving a high degree of primary implant stability (high value of insertion torque).

- Where clinical judgment is that immediate loading is appropriate, and patient desire to shorten the treatment period and avoid an extended period of edentulism is expressed, immediate loading of dental implants is an acceptable alternative to conventional protocols.

1. Branemark PI, Hansson $\mathrm{BO}$, Adell R, et al. Osseointegrated implants in the treatment of edentulous jaw. Experience from a 10-year period. Scand J Plast Reconstr Surg Suppl 1977; 16: 1-132.

2. Esposito M, Worthington HV, Coulthard P. Interventions for replacing missing teeth: different times for loading dental implants. Cochrane Database Syst Rev 2003; issue 1.

3. Esposito M, Grusovin MG, Achille H, Coulthard P, Worthington HV. Interventions for replacing missing teeth: different times for loading dental implants. Cochrane Database Syst Rev 2009; issue 1.

4. Thorlund K, Imberger G, Walsh M, et al. The number of patients and events required to limit the risk of overestimation of intervention effects in meta-analysis a simulation study. PLoS One 2011; 6: e25491.

Evidence-Based Dentistry (2013) 14, 109-110. doi:10.1038/sj.ebd.6400967 